\section{Bevacizumab - der neue Standard in der Darmkrebstherapie}

\begin{abstract}
Die Europäische Arzneimittelagentur EMEA hat eine breite Zulassungserweiterung für Bevacizumab $\left(\right.$ Avastin $\left.^{\circledR}\right)$ in der Therapie des metastasierten kolorektalen Karzinoms ( $m C R C$ ) erteilt: Ab sofort kann die Anti-VEGF-Therapie mit Bevacizumab in Kombination mit allen Fluoropyrimidin-basierten Chemotherapien inklusive Oxaliplatin und Capecitabin $\left(X_{\text {eloda }}^{\circledR}\right)$ beim $m C R C$ eingesetzt werden. Bevacizumab bildet damit die Grundlage aller Regime zur First-Line-Therapie des mCRC.
\end{abstract}

Die Zulassung basiert auf den Daten der Studie NO16966. In der internationalen Studie konnte gezeigt werden, dass die zusätzliche Gabe von Bevacizumab first-line $(7,5 \mathrm{mg} / \mathrm{kg}$, alle 3 Wochen, für 6 Monate) zu den Chemotherapie-Regime XELOX (Capecitabin $1000 \mathrm{mg} / \mathrm{m}^{2}$, zweimal täglich oral, Tag 1-14, alle 3 Wochen, plus Oxaliplatin $130 \mathrm{mg} / \mathrm{m}^{2}$, i. v.) oder FOLFOX-4 (5-FU/FS-Dauerinfusion plus Oxaliplatin) zu einer signifikanten Verlängerung des progressionsfreien Überlebens (PFS) von 8,0 auf 9,4 Monate führte $(\mathrm{p}<0,0023)$. Wurde Bevacizumab über 6 Monate hinaus, bis zum Tumorprogress gegeben, konnte das PFS im Vergleich zur alleinigen Chemotherapie sogar um mehr als $30 \%$ (10,4 versus 7,9 Monate; $\mathrm{p}<0,0001$ ) gesteigert werden [1] (Abb. 1). Es wurden keine neuen oder unerwarteten Nebenwirkungen für Bevacizumab beobachtet. Daten der ersten Zulassungsstudie von Hurwitz et al. [2], in der Bevacizumab first-line mit 5-FU/FS plus Irinotecan kombiniert wurde, was zu einem PFS von 10,6 Monaten
Die NO16966-Studie bestätigt damit die führte, gegenüber 6,2 Monaten unter der alleinigen Chemotherapie $(\mathrm{p}<0,00001)$. In der Hurwitz-Studie wurde die Bevacizumab-Therapie großteils bis zum Tumorprogress und damit fast 3 Monate länger als in der Studie NO16966 fortgesetzt. Das Gesamtüberleben der Patienten konnte erstmalig um etwa 5 Monate auf 20,3 Monate verlängert werden.

\section{Tägliche Praxis bestätigt Studienergebnisse}

Dass sich die guten Daten zur ÜberlebensverAlltag zeigen lassen, machen die beiden großen Beobachtungsstudien First BEAT und BRiTE deutlich. First BEAT wurde mit 1914 Patienten aus 41 Ländern - außer USA durchgeführt, die Bevacizumab in der FirstLine-Therapie, kombiniert mit einer Standard-Chemotherapie nach Wahl, erhielten. Das vorläufige mediane PFS liegt auch hier für alle Kombinationschemotherapien bei knapp 11 Monaten [3]. An der BRiTE-Studie nahmen knapp 2000 US-amerikanische Patienten teil. Das mediane PFS im Gesamtkollektiv betrug 10,2 Monate. Es wurde ein medianes Gesamtüberleben von 27,1 Monaten erreicht [4]. Das sind die längsten Überlebenszeiten, die bei Patienten mit mCRC je beobachtet wurden.

\section{Bevacizumab ermöglicht kurative Metastasenresektion}

Die First-BEAT-Beobachtungsstudie konnte außerdem zeigen, dass Bevacizumab first-line längerung mit Avastin auch im klinischen

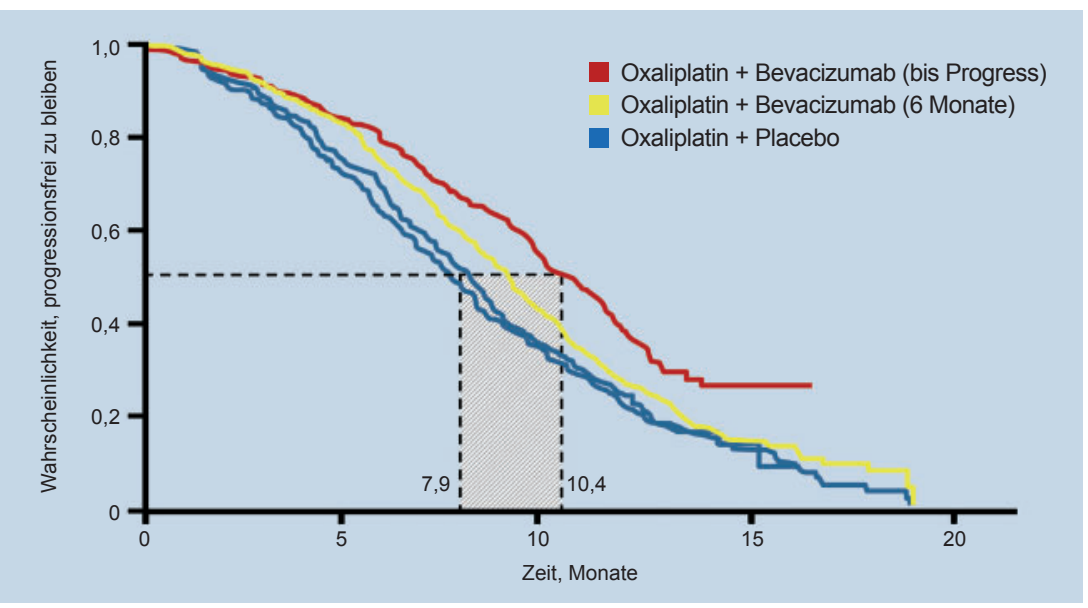

Abb. 1. Durch Behandlung bis zum Progress kann das PFS auf über 10 Monate verlängert werden. in Kombination mit einer Standard-Chemotherapie die wirksame und sichere kurative Resektion initial inoperabler Metastasen ermöglicht. Eine vollständige Metastasenresektion (R0-Resektion) war demnach bei rund $77 \%$ der operierten Patienten möglich, obwohl diese vor dem Beginn der Therapie als inoperabel eingestuft wurden. Von den 1914 in der derzeitigen Datenanalyse erfassten Patienten konnten 225 (12\%) im Laufe der Behandlung mit kurativem Ziel operiert werden. Eine vollständige Entfernung der Metastasen wurde bei 173 (9\%) der Patienten erreicht. Die besten Ergebnisse erzielten die Patienten, deren Metastasen sich ausschließlich auf die Leber beschränkten $(n=704)$. Von ihnen wurden $15,2 \%$ operiert, und eine R0-Resektion konnte bei 85 Patienten $(12,1 \%)$ durchgeführt werden. Wurde Bevacizumab mit einem Oxaliplatin-haltigen Regime kombiniert, war eine Resektion der Lebermetastasen sogar bei $20,3 \%$ und eine R0-Resektion bei $15,4 \%$ der Patienten möglich [5]. Das hohe Sicherheitsprofil früherer Kontrollen wurde bestätigt, es wurde kein Anstieg der Wundheilungskomplikationen oder Blutungsereignisse beobachtet. Damit übertreffen die Erfolge der Therapie mit Bevacizumab in der breiten klinischen Anwendung alle anderen bisher in Studien mit «Targeted therapies»/Chemotherapie-Kombinationen dokumentierten Ergebnisse und bestätigen den Einsatz von Bevacizumab als neuen Standard in der Darmkrebstherapie.

\section{Referenzen}

1 Saltz et al. ASCO GI 2007, \#238

2 Hurwitz et al. N Engl J Med 2004; 350:2335-42

3 Berry et al. ASCO GI 2008, \#350

3 Berry

5 Cunningham et al. ASCO GI 2008, \#44

Impressum

Bevacizumab - der neue Standard in der Darmkrebstherapie

PharmaForum in OnKologie 31 | 4 | 08

(c) 2008 by S. Karger Verlag für Medizin und Naturwissenschaften $\mathrm{GmbH}$

Lörracher Straße 16a

79115 Freiburg, Deutschland

Mit freundlicher Unterstützung durch Roche Pharma AG.

Die Wiedergabe von Gebrauchsnamen, Handelsnamen, Warenbezeichnungen usw. in dieser Zeitschrift berechtigt auch ohne besondere Kennzeichnung nicht zur Annahme, dass solche Namen im Sinne der Warenzeichen- und Markenschutz-Gesetzgebung als frei zu betrachten wären und daher von jedermann benutzt werden dürfen.

Der Verlag und die Herausgeber der Zeitschrift übernehmen keine Verantwortung für diese Rubrik. 